\title{
Millimeter-Wave Phaseless Antenna Measurement Based on a Modified Off-Axis Holography Setup
}

\author{
Ana Arboleya ${ }^{1}$ (D) . Juha Ala-Laurinaho² . \\ Jaime Laviada ${ }^{1}$ Yuri Álvarez ${ }^{1}$. \\ Fernando Las-Heras ${ }^{1}$ • Antti V. Räisänen ${ }^{2}$
}

Received: 28 August 2015 / Accepted: 29 September 2015/

Published online: 21 October 2015

(C) The Author(s) 2015. This article is published with open access at Springerlink.com

\begin{abstract}
A novel scheme for planar near-field phaseless antenna measurement based on off-axis holography is presented. Separation of the image terms of the hologram is artificially increased by multiplexing the measurements of two sub-sampled holograms generated with two $180^{\circ}$ phase-shifted reference waves. Combination of both sub-sampled holograms produces replicas of the image terms at half a period distance of the originals in the spectral domain, while the amplitude of the original image terms is highly reduced, easing the filtering process of the desired replica. The higher separation of the image terms reduces overlapping making the method suitable also for the characterization of medium and low gain antennas in the near-field. As the separation is artificially increased, the reference antenna can be placed close to the antenna under test allowing to reduce the scan distance and the sensitivity to scan axis errors. Nevertheless, spatial multiplexing requires the retrieved data to be spatially low-pass filtered to remove the effect of the aliasing. Mirror reflection is used for illuminating the acquisition plane with the reference wave, being the phase shift achieved by means of a mechanical displacement of the mirror. The effect of the location of the reference antenna on the position and shape of the image terms and their replicas has been studied through numerical simulations for a setup in the W-band. Experimental validation of the method is presented for the characterization of three different antennas at $94 \mathrm{GHz}$.
\end{abstract}

Ana Arboleya

aarboleya@tsc.uniovi.es

1 Departamento de Ingeniería Eléctrica, Universidad de Oviedo, 33203 Gijón, Spain

2 Department of Radio Science and Engineering and MilliLab, Aalto University, 00076 Espoo, Finland 
Keywords Phaseless · Antenna measurement - Diagnostics · Near-field · Off-axis · Holography $\cdot$ Millimeter-wave

\section{Introduction}

Characterization of antennas in near-field (NF) involves the acquisition of amplitude and phase information so the far-field (FF) radiation pattern or the aperture fields for antenna diagnostics can be obtained after post-processing the complex acquired data. Nevertheless, phase acquisition, fundamentally at mm- and submm-wave bands, is a very challenging and expensive process due to the need of keeping a stable phase reference which demands not only a very stable source but also steady temperature conditions and high mechanical accuracy [1-4].

The aforementioned difficulties of the phase measurement process have motivated the development of the so-called phaseless techniques, the interferometric technique being one of the most employed methods to retrieve the phase of the antennas. This technique, based on recording the intensity pattern formed by the field of the antenna under test (AUT) and the field of a reference antenna, which is previously known in amplitude and phase, as introduced by Gabor in the optics field [5], and then modified to use an off-axis reference by Leith and Upatnieks [6], is often refered to as off-axis indirect holography. Holography was first used for antenna metrology by Bennett [7] and Anderson [8].

The off-axis technique relies on the filtering of one of the terms of the hologram in the $k$-space, whose position depends on the angle formed by the reference antenna and the acquisition plane [4]. In conventional setups (see Fig. 1a), this reference antenna has to be placed far from the AUT in order to illuminate the acquisition plane with a plane wave. This leads to bigger dimensions of the setup (scan plane distance and size) and higher sensitivity to scan axis errors [4, 9]. Another option to create the plane wave reference is to use a shaped, e.g., parabolic mirror. This requires accurate and expensive machining. Also, the reflector could then be used directly as a collimator in a compact antenna test range setup [10]. Some numerical experiments have demonstrated the advantages of using spherical wave-fronts as reference signals for the characterization of highly directive antennas [4].

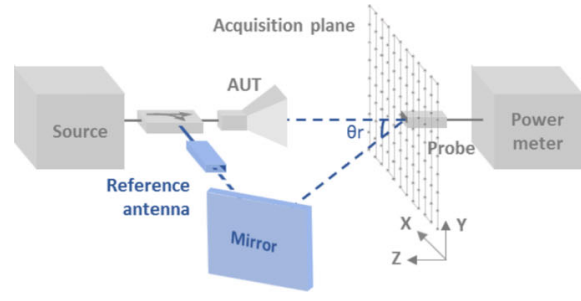

(a)

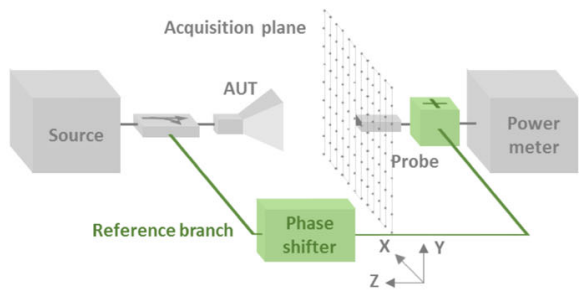

(b)

Fig. 1 Schemes for the holography setups. a Conventional setup with mirror reflection of the reference antenna and $\mathbf{b}$ synthesized reference antenna setup 
Further development of the conventional off-axis setup has been done to achieve better results in the filtering process. The use of the so called modified holograms without the auto-correlation terms allows for a reduction of the separation between the AUT and the reference antenna, and consequently, for a reduction of the sampling rate. This enables to lower the scan distance and the sensitivity to error positioning of the probe antenna [4]. However, an extra measurement for the independent characterization of the intensity of the AUT is needed in order to subtract its effect from the complete hologram.

Substitution of the radiated reference wave by a synthesized one allows to control the phase shifts and, therefore, the separation of the cross-correlation terms, although a denser sampling is needed in order to extend the frequency spectrum domain [11, 12]. However, the cited works have been validated in the $X$-band and they are not likely to be used at higher frequency bands wherein the connection between the directional coupler and the moving probe (see Fig. 1b) should be made with waveguide components.

It is possible to avoid the use of phase shifters by implementing three-dimensional scans to mechanically introduce the phase shifts [13], although the majority of planar measurement ranges cannot perform this type of acquisition, as they are limited to plane acquisitions.

In this contribution, a novel setup in which the separation between the crosscorrelation terms of the hologram is artificially increased by combining two subsampled holograms with a phase difference of $180^{\circ}$, is proposed. The desired phase difference is produced by a small movement of the flat reference wave mirror shown in Fig. 1a. Hence, although the sampling rate has to be slightly increased due to the wider spatial bandwidth of the acquired interferometric signal in the $k$-space, it is possible to work with the complete hologram. This avoids the need of an additional measurement for AUT characterization to subtract its auto-correlation term, and makes possible to place the reference antenna close to the AUT as in the modified holograms setups. Diminishing the size of the setups overcomes the problems associated to the use of large acquisition planes [4].

This bigger separation of the image terms of the hologram eases the filtering process allowing to reduce the error of the phase retrieval with respect to the conventional off-axis holography method. Thanks to this, it is possible to use the proposed method for the characterization of medium and low gain antennas, with wider spectra, although, as it will be shown in Section 4.2, the results are also improved for the case of measuring high gain antennas.

As a counterpart, due to the sub-sampling, a low-pass spatial filtering is needed in order to correctly retrieve the signal of the AUT, affected by aliasing, as well as a correction of the level of the retrieved amplitude.

\section{Proposed Method}

In off-axis holography, a hologram, $H(\vec{r})$, is obtained after squaring the sum of an intensity pattern created by the field components of the AUT, $E_{a u t}(\vec{r})$, and the reference antenna, $E_{r}(\vec{r})$, acquired by the probe. As the amplitude of the reference antenna 
is previously known, its effect is removed directly from the hologram, given by the following expression

$$
\begin{aligned}
H(\vec{r}) & =\left|E_{\text {aut }}(\vec{r})+E_{r}(\vec{r})\right|^{2}-\left|E_{r}(\vec{r})\right|^{2} \\
& =\left|E_{\text {aut }}(\vec{r})\right|^{2}+E_{\text {aut }}(\vec{r}) E_{r}^{*}(\vec{r})+E_{\text {aut }}^{*}(\vec{r}) E_{r}(\vec{r})
\end{aligned}
$$

where $\vec{r}$ defines the position of the probe and ( $)^{*}$ indicates complex conjugate.

After Fourier transforming to the $k$-space, the hologram, $h(\vec{k})$, is given by

$$
h(\vec{k})=\widetilde{H(\vec{r})}=\left|\widetilde{E_{a u t}(\vec{r})}\right|^{2}+\widetilde{E_{a u t}(\vec{r})} \otimes \widetilde{E_{r}^{*}(\vec{r})}+\widehat{E_{a u t}^{*}(\vec{r})} \otimes \widetilde{E_{r}(\vec{r})}
$$

being $(\widetilde{)})$ the Fourier Transform (FT) and $\otimes$ the convolution operator. The first term is called the auto-correlation term and the following two, containing information about the amplitude and phase of the AUT, are known as cross-correlation or image terms.

The separation of the cross-correlation terms in the $k$-space can be controlled with the off-axis angle of the reference antenna, $\theta_{r}$ (see Fig. 1a). In particular, the center of the cross-correlation terms is located at

$$
k_{r, x}= \pm \Delta \phi / \Delta x= \pm k_{0} \sin \theta_{r}
$$

considering the reference antenna is placed with the offset in the $x$-axis [14]. In the previous formulation, $\Delta \phi$ is the phase difference between consecutive samples of the reference signal in the acquisition plane, $\Delta x$ is the sampling rate, and $k_{0}$ is the wavenumber.

If the separation of the terms is enough to avoid overlapping, the image term $\left.\widehat{\left(E_{\text {aut }}(\vec{r})\right.} \otimes \widehat{E_{r}^{*}(\vec{r})}\right)$ can be filtered and the complex magnitude of the AUT can be retrieved back in the spatial domain, after dividing by the expression of the conjugate of the reference antenna.

According to [2], the spatial bandwidth for the radiated field for a planar acquisition can be considered as $W=k_{0}$. Hence, as it is shown in Fig. 2a, to avoid overlapping with a conventional setup, $k_{r} \geq 3 k_{0} / 2$.

Nevertheless, for a radiated reference wave, the maximum offset angle is restricted to $90^{\circ}$ and, therefore, $k_{r, \max }=k_{0}=2 \pi / \lambda[15]$. This separation may not be enough to avoid the overlap between the aforementioned spectral components in (2) (see Fig. 2a for a graphical description), specially when working in the NF with medium and low gain antennas which have wider spectra. Besides, to illuminate the acquisition plane with a plane wave, as it is usually done in conventional setups, the reference

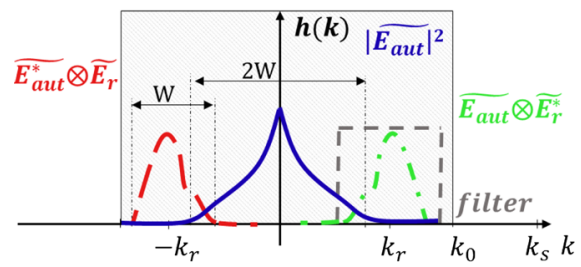

(a)

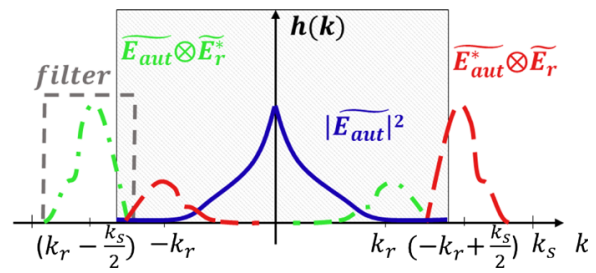

(b)

Fig. 2 Spectrum of the hologram. a Conventional setup and $\mathbf{b}$ proposed setup with the replicas and the original terms. The central shaded box indicates the visible part of the spectrum 
antenna has to be placed far away from the AUT, being also necessary to increase the separation and the size of the acquisition plane, which makes the setup more sensitive to scan axis errors [4].

The use of synthesized reference waves removes the offset angle limitation and makes possible to displace the cross-correlation terms to the non-visible part of the $k$-space $\left(k> \pm k_{0}\right)$, providing a denser sampling rate is used to extend its limits, defined by $k_{s}= \pm \pi / \Delta x[12,16]$.

The proposed setup is an intermediate solution to artificially increase the separation of the cross-correlation terms while using a radiated reference wave. The method relies on the spatial multiplexing of two sub-sampled holograms. The first hologram is recorded from the initial position of the the mirror and its samples are stored in the even columns of the final hologram. The second hologram, stored in the odd columns, is recorded after introducing a mechanical displacement of $\lambda / 2$ in the mirror position (see Fig. 3b) which produces a phase shift of $180^{\circ}$ in the phase of the reference wave in the acquisition plane, while leaving the amplitude practically unchanged. After that, a hologram is composed by interleaving the columns, i.e., the samples acquired along the y-axis, of the two measured holograms (see Fig. 3a).

When working with the two sub-sampled reference signals, two replicas at $k_{s} / 2$ of the original cross-correlation terms appear, so that the hologram has five terms, the auto correlation term, the two image terms, and the two replicas of the image terms as schematically shown in Fig. 2 b.

If the reference signal is a plane wave, due to the phase-shift between the signals, the original cross-correlation terms are canceled while the amplitude of the replicas is reinforced (see Appendix) allowing to easily filtering one of that replicas. Nevertheless, for spherical wave fronts, as the phase difference between consecutive samples of the signal is not constant in the acquisition plane, the original cross-correlation terms are not completely canceled, being this the main drawback of using spherical wave fronts as reference signal. This will be analyzed in the Numerical simulations section.

Nonetheless, the use of spherical reference wave fronts as reference signals has many advantages such as the reduction of the scan plane size and distance to the AUT, and the reduction of the distance between the AUT and the reference antenna. This reduction in size of the setup, as stated in [4], helps to reduce the scan errors and the sensibility of the system.

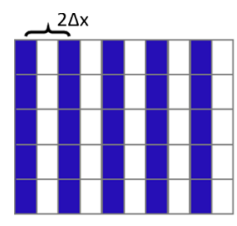

Subsampled hologram 1

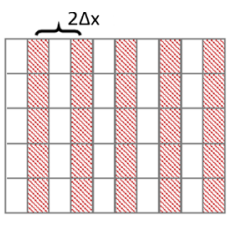

Subsampled hologram 2

(a)

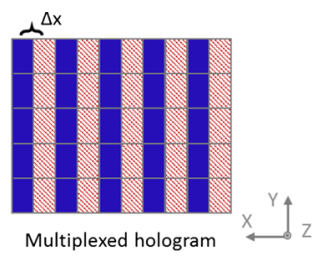

position 1

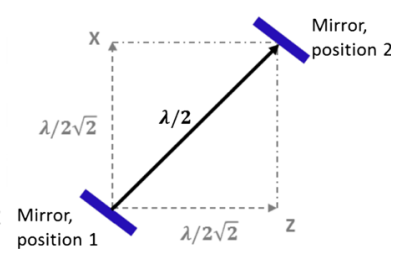

(b)

Fig. 3 Hologram recording method. a Hologram composition by multiplexing data from the two subsampled holograms and $\mathbf{b}$ mirror displacement for the $180^{\circ}$ phase shift in the second hologram samples 
Other advantages of using a spherical reference wave front are the possibility of controlling the scan plane edge illumination, being able to lower the reference onaxis sidelobes, and the reduction of the dynamic range needed in the $k$-space as the cross-correlation terms no longer have the shape of the AUT spectrum but a narrower one [4].

However, as the filtered term corresponds to the replica of the image term, the retrieved signal back in the spatial domain is contaminated with high-frequency noise. In order to correctly retrieve the AUT signal, a low-pass filtering process is necessary after the phase retrieval process.

Thanks to the aforementioned advantages, it is possible to reduce the error of the phase-retrieval with respect to the conventional setup as it will be shown in the numerical and experimental validation sections.

\section{Numerical Simulations}

In order to study the influence of the type of the reference wave front in the cancellation of the original image terms of the hologram and the shape and position of its replicas, the setup is first simulated, using MATLAB, for three different reference fields.

The conventional setup is simulated first by keeping the mirror fixed for the whole acquisition of the hologram with a sampling rate of $\lambda / 6$, while the proposed method is implemented by multiplexing two sub-sampled holograms corresponding to the two different positions of the mirror, each of them with a sampling rate of $\lambda / 3$ to compose a final hologram also with a sampling rate of $\lambda / 6$. For both cases, the extension of the $k$-space is $k_{s}= \pm \pi / \Delta x= \pm 3 k_{0}$. The method is also studied for the case of multiplexing two plane waves instead of the spherical waves with a phase shift of $180^{\circ}$ and the same amplitude than the spherical ones, to verify the correct cancellation of the aforementioned terms of the spectra.

The AUT is a vertically polarized rectangular aperture of $2.5 \lambda$ by $2 \lambda$ at $94 \mathrm{GHz}$. A WR10 pyramidal horn, placed at $100 \lambda$ from the AUT in the $x$-axis and $20 \lambda$ in the $\mathrm{Z}$-axis, is used as a reference antenna. The acquisition plane is $160 \lambda$ by $160 \lambda$ placed at a distance of $z_{0}=50 \lambda$ of the AUT aperture. And the mirror is placed at $20 \lambda$ from the reference antenna with a $45^{\circ}$ tilt in the $x$-axis.

The phase difference between consecutive samples along the $x$-axis is shown in Fig. 4a for the first position of the mirror. This phase difference is not shown for the second position of the mirror since it is identical. In order to simulate the plane-waves, they are given an angle so that the phase difference between consecutive samples is $45^{\circ}$, which is the mean phase difference of the previously described spherical waves.

With the obtained phase and according to Eq. 3, the cross-correlation terms are centered in $k_{r}= \pm 0.75 k_{0}$, and the desired replicas placed at a distance of $3 k_{0}$ of the originals, that is at $k_{r_{\text {replicas }}}= \pm 0.75 k_{0} \mp 3 k_{0}=\mp 2.25 k_{0}$.

Figure $4 \mathrm{~b}$ shows the spectrum of the reference antenna for the three studied cases. The spectrum of the reference wave for the conventional setup (dashed line) is formed of just one term centered in $0.75 k_{0}$. The image terms of the hologram are in the same 


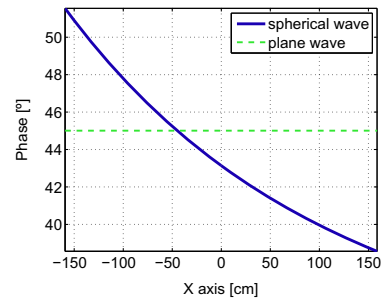

(a)

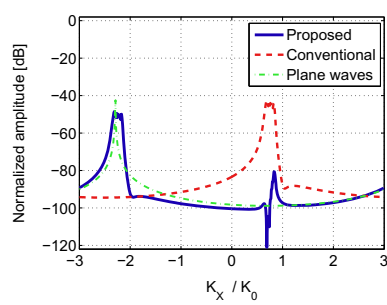

(b)

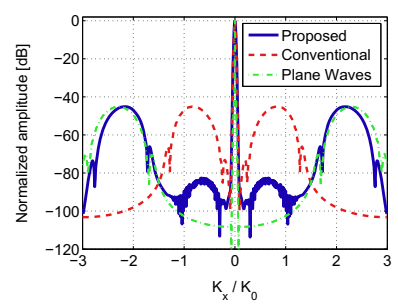

(c)

Fig. 4 Simulation results. a Phase slope of the reference waves, $\mathbf{b} \times$ cut $(y=0)$ of spectrum of the reference signal for the case of using spherical waves for the proposed and conventional setups and for the case of using plane waves, and $\mathbf{c}$ spectrum of the holograms for the different reference waves and setups

position (Fig. 4c) and depending on the spectral width of the AUT, might overlap with the auto correlation term introducing error in the filtering process.

The dotted-dashed line in Fig. 4b shows the spectrum of the reference wave in case of having two ideal plane waves with a $180^{\circ}$ phase shift multiplexed. For this case, as it is demonstrated on the Appendix, the original image terms are canceled due to the phase shift, while the amplitude of the replicas, at $\mp 2.25 k_{0}$, is reinforced. This would be the ideal situation, as the separation of the image terms of the hologram with the auto correlation term is maximized (see Fig. 4c).

Finally for the proposed setup (solid line in Fig. 4b), the original term at $0.75 k_{0}$ is not completely canceled, whereas the replica appears in $-2.25 k_{0}$ as expected. It is important to note that the spectral power density of the signal is distributed between the replica and the original term, i.e., the replica has lower amplitude than the image term for the conventional setup and this will affect the amplitude retrieval of the AUT and will have to be corrected, as it will be shown in the next section. The effect of the partial cancellation of the original terms can be seen in the hologram, Fig. 4c.

Although the original image terms are not completely canceled in the proposed setup, Fig. 4c shows that the overlapping between the replicas and the original image terms is less than the overlapping of the image terms and the auto correlation term in the conventional setup. This will be more clearly seen in the measurement results section wherein the noise and the non-ideal acquisition environment will deteriorate the quality of the holograms.

Besides, the use of spherical waves has many other advantages that have been previously mentioned such as the reduction of the size of the setup, lowering the scan errors and the sensitivity of the system, and the possibility of narrowing the spectrum of the image terms of the hologram according to the shape of the spectrum of the reference antenna [4], as it can be seen in Fig. 4c if the width of the replicas of the proposed setup is compared to the one obtained when considering plane waves.

\section{Experimental Validation}

In order to validate the proposed method, a measurement setup for phaseless antenna characterization in the $\mathrm{W}$ band has been implemented. 
The measurements have been made in a planar measurement range. Three different antennas with different directivity have been studied at $94 \mathrm{GHz}$ : A $64 \mathrm{~mm}$ circular lens antenna of $25 \mathrm{~dB}$ gain, feeded with a $10 \mathrm{~mm}$ length open ended waveguide (OEWG) antenna, a pyramidal horn antenna of $20 \mathrm{~dB}$ gain, and an OEWG antenna of $6 \mathrm{~dB}$ gain.

The reference antenna, another pyramidal horn antenna, is placed at $120 \mathrm{~mm}$ of the feeding point of the AUT and its radiation is directed towards the acquisition plane, at $125 \mathrm{~mm}$ of the aperture of the AUT by means of mirror reflection.

The element used as a mirror is a smoothened metallic plate of $290 \mathrm{~mm}$ by $221 \mathrm{~mm}$ with a $15^{\circ}$ tilt in the $x$-axis, whose edges are surrounded by electromagnetic absorber. The details of the setup for the three different antennas are shown in Fig. 5.

\subsection{Amplitude Correction}

Due to the fact that only a fraction of the spectral density of the hologram is filtered during the phase retrieval, a small correction in the retrieved amplitude of the AUT also needs to be made. The power spectral density of the reference signal is distributed between the replica and the not completely canceled image term (Fig. 3b). This way, when filtering only the replica, not all the power is taken into account and the signal transformed back to the spatial domain has lower amplitude than the original. Therefore, prior to the hologram calculation, it is necessary to study the spectrum of the reference antenna independently, in order to obtain the correction factor to apply to the retrieved amplitude of the AUT.

To do that, the spectrum of the main component of the reference antenna, $E_{r}(\vec{r})$, which is characterized in amplitude and phase, is obtained as

$$
e_{r}(\vec{k})=\widetilde{E_{r}}(\vec{r})
$$

The part corresponding to the replica is filtered with the same window that will be used in the phase retrieval process, a rectangular window $\Pi\left(k_{1}, k_{2}\right)$ defined from

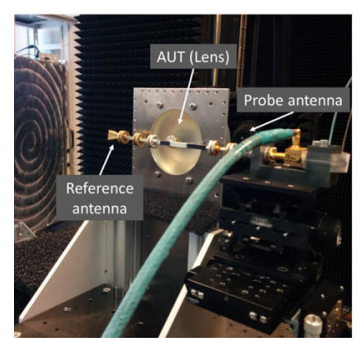

(a)

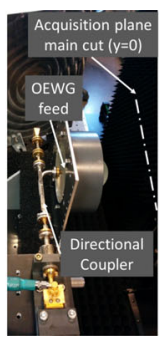

(b)

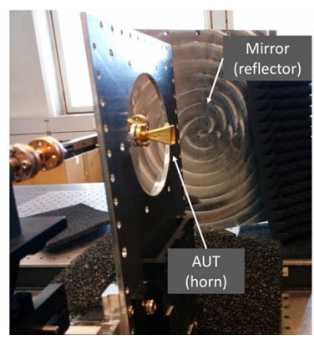

(c)

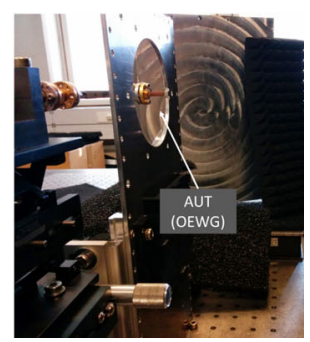

(d)

Fig. 5 Measurement setup for the setup validation at $94 \mathrm{GHz}$ before placing the electromagnetic absorber. $\mathbf{a}$ Rear view of the setup for the lens antenna, $\mathbf{b}$ top view of the setup for the lens antenna, $\mathbf{c}$ front view of the setup for the horn antenna, and $\mathbf{d}$ front view of the setup for the OEWG antenna 


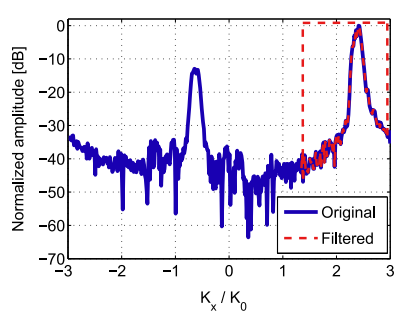

(a)

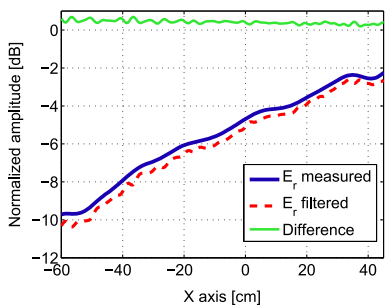

(b)

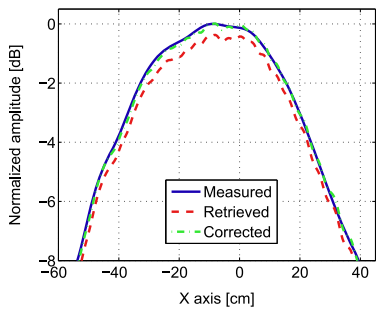

(c)

Fig. 6 Analysis of the horn reference antenna for the amplitude correction of the retrieved signal. a Spectrum of the reference signal, $\mathbf{b}$ detail of the normalized amplitude of the measured reference antenna compared to its amplitude after filtering in the spectral domain, and c detail of the amplitude correction of the retrieved signal of the AUT for a horn antenna

$k_{1}$ to $k_{2}$, as shown in Fig. 6a for the horn antenna used as reference antenna in this measurement example.

$$
e_{r_{\text {filtered }}}(\vec{k})=e_{r}(\vec{k}) \Pi\left(k_{1}, k_{2}\right)
$$

Then the filtered signal is transformed back to the spatial domain by means of an inverse FT denoted by $\tilde{(\cdot)}^{-1}$ as indicated in Eq. 6, and its amplitude is compared to the measured amplitude, obtaining the correction factor, $\mathrm{CF}$, defined in Eq. 7.

$$
\begin{gathered}
E_{r_{\text {filtered }}}=\left({ }_{e_{r_{\text {filtered }}}}\right)^{-1} \\
C F[d B]=E_{r}(\vec{r})[d B]-E_{r_{\text {filtered }}}(\vec{r})[d B]
\end{gathered}
$$

It is important to note that the correction factor is only dependent on the reference antenna, for this case the mean of CF is $0.38 \mathrm{~dB}$ as it is shown in Fig. 6b. The correction is shown for the case of applying the correction factor to the horn antenna as an AUT in Fig. 6c.

\subsection{Measurement Results}

Both phaseless setups, the conventional and the proposed one, have been implemented in order to compare their performance for the three different types of antennas. A direct acquisition of amplitude and phase has also been made with the

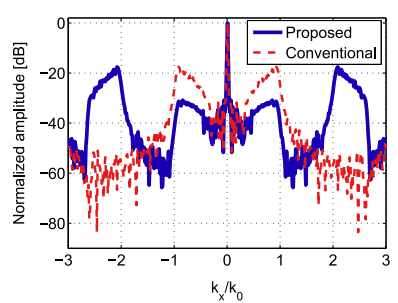

(a)

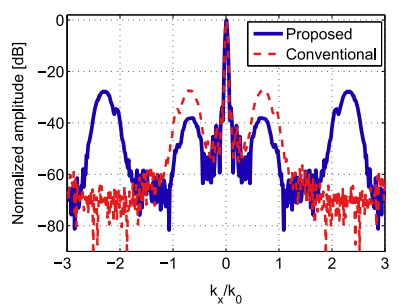

(b)

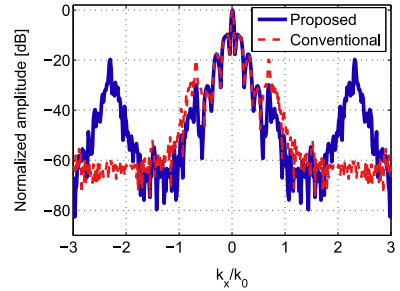

(c)

Fig. 7 Spectrum of the holograms for the proposed and conventional setups. a OEWG antenna, b horn antenna, and $\mathbf{c}$ lens antenna 
purpose of using it as reference and quantify the error of the phase retrieval methods (see Eq. 8). All the results are shown for an x-cut of the AUT ( $y=0)$, which is horizontally polarized.

First step of the phase retrieval algorithm is the calculation of the spectrum of the hologram as defined in Eq. 2, to filter the desired image term (or replica). The hologram spectra are shown for both setups in Fig. 7. The filter extension for the conventional setups goes from $-2 k_{0}$ to $-0.3 k_{0}$ approximately, while the one used in the proposed setups goes from $-3 k_{0}$ to $-k_{0}$.

It is clearly seen that the image terms are wider for less directive antennas, Fig. 7a, $\mathrm{b}$, and in the case of the conventional setup, they overlap with the auto correlation term, introducing error in the filtering process, while for the proposed setup, the position of the replica and the amplitude reduction of the original image terms, ease the filtering process allowing to correctly retrieve the signal.

Directive antennas, despite having narrower image terms, have wider auto correlation terms, Fig. 7c, therefore, unless special care is given to leveling the AUT and

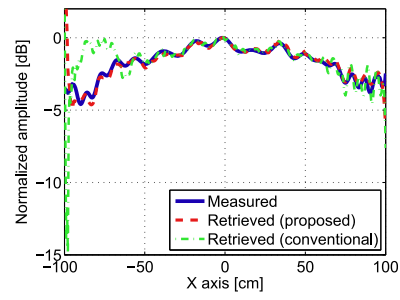

(a)

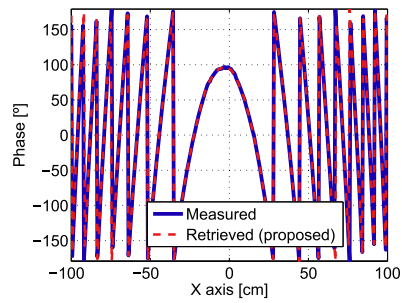

(d)

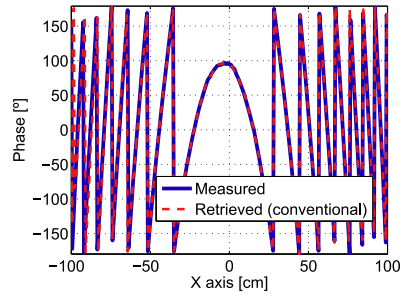

(g)

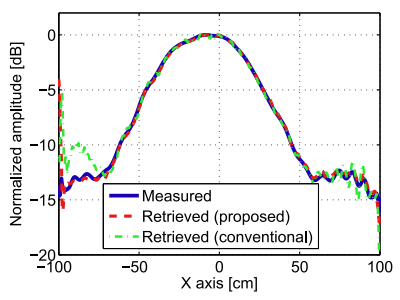

(b)

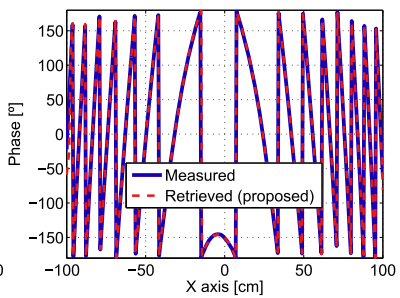

(e)

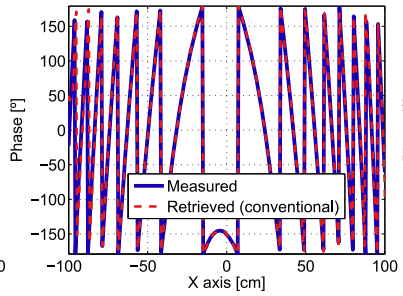

(h)

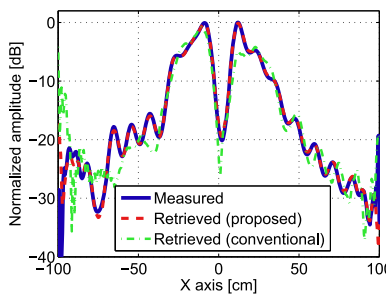

(c)

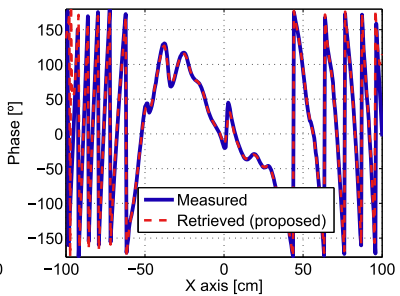

(f)

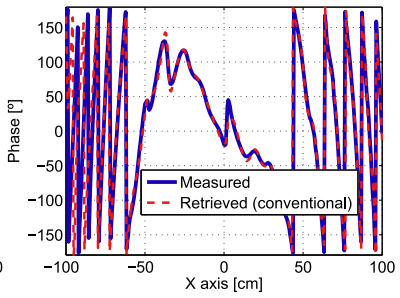

(i)

Fig. 8 Retrieved fields compared to the direct acquisition for the proposed and conventional setups. a Amplitude of the OEWG, $\mathbf{b}$ amplitude of the horn, $\mathbf{c}$ amplitude of the lens, $\mathbf{d}$ phase of the OEWG, proposed setup, e phase of the horn, proposed setup, $\mathbf{f}$ phase of the lens, proposed setup, $\mathbf{g}$ phase of the OEWG, conventional setup, $\mathbf{h}$ phase of the horn, conventional setup, and $\mathbf{i}$ phase of the lens, conventional setup 
the reference antenna branches by adding attenuators or amplifiers, the overlapping of the terms of the hologram will be very high for the conventional setup. With the proposed setup, it is still possible to correctly filter the desired term without the need to resort to auxiliary components to level the branches.

Once the hologram has been filtered, the amplitude and phase of the AUT can be retrieved after transforming the filtered data back to the spatial domain and compensating for the effect of the reference signal. In case of the proposed setup, the amplitude correction method and a low pass filter to eliminate the ripple introduced by the aliasing have to be applied.

The retrieved amplitude for the conventional and proposed setups is compared to the direct measured amplitude for the three antennas in Fig. 8a-c. With the proposed setup, the error is lower on the edges of the acquisition plane due to the overlapping reduction of the spectral terms. The retrieved phase is also shown, compared to the directly measured one, in Fig. 8d-f for the proposed setup and in Fig. 8g-i for the conventional setup.

As the error of the retrieval process is reduced on the edges of the acquisition plane, another advantage of the proposed setup is that the valid angular margin of the NF-FF transformation, depending on the size of the acquisition plane, is also increased in case the data have to be transformed to FF.

To quantify the quality of the phase retrieval method, the following error metric is defined:

$$
e[\%]=100 \frac{\left|E_{\text {meas }}-E_{\text {ret }}\right|}{\left|E_{\text {meas }}\right|}
$$

where $E_{\text {meas }}$ is the measured field ( in amplitude and phase), and $E_{\text {ret }}$ is the retrieved field with the off-axis holography method. The error is calculated for the three antennas in case of using both setups and depicted in Fig. 9 where the better performance of the proposed method is clearly observed for the whole acquisition plane, but mainly on the edges.

As the error of the phase retrieval seems to be higher in those areas corresponding to low amplitude levels of the AUT, a weighted mean error is defined as follows:

$$
e_{w}[\%]=\frac{1}{N} \sum_{i=1}^{N}|e[\%](i)|\left|E_{r e t}(i)\right|
$$

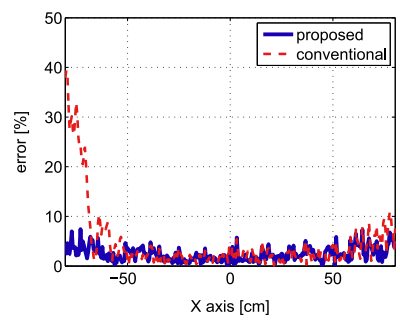

(a)

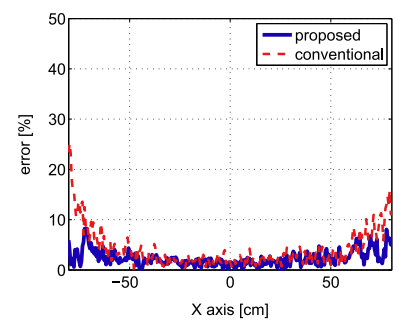

(b)

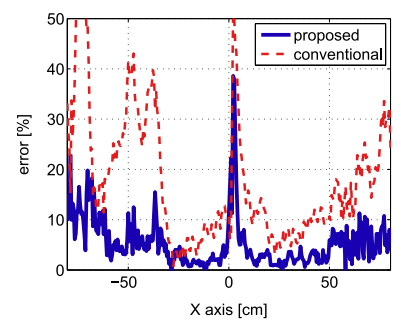

(c)

Fig. 9 Error of the phase retrieval process for the proposed and conventional setups. a OEWG antenna, b horn antenna, and $\mathbf{c}$ lens antenna 
Table 1 Mean error of the phase retrieval technique for the conventional and the proposed methods for the three studied antennas

\begin{tabular}{llllll}
\hline & \multicolumn{2}{l}{ Conventional setup } & & \multicolumn{2}{l}{ Proposed setup } \\
\cline { 2 - 3 } \cline { 5 - 6 } & Mean $(\%)$ & Weighted mean $(\%)$ & & Mean $(\%)$ & Weighted mean $(\%)$ \\
\hline OEWG & 4.64 & 3.62 & 2.41 & 2.04 \\
Horn & 4.13 & 1.64 & 2.31 & 1.03 \\
Lens & 17.91 & 3.10 & 5.38 & 0.87 \\
\hline
\end{tabular}

Both types of error are gathered in Table 1. The high error in the central part of the acquisition plane for the lens is due to the null in the amplitude and it is compensated with the use of the weighted mean error. As it is observed, the performance of the proposed setup is better for all the studied cases, regardless of the type and directivity of the antennas.

\section{Conclusions}

A novel setup based on off-axis holography is proposed. By means of multiplexing two subsampled holograms with a $180^{\circ}$ phase shift, it is possible to achieve larger separation of the image terms while keeping the reference antenna close to the AUT.

For the validation of the setup, mirror reflection has been used to illuminate the acquisition plane with the reference wave, and the phase shift of the reference signal has been obtained by introducing a mechanical displacement of the mirror for the acquisition of the second subsampled hologram.

As the separation of the image terms, replicas in this case, is larger than for the conventional setup, the proposed method is suitable for low and medium directive antenna measurement. Furthermore, it has also been demonstrated that the proposed method also improves the results for directive antennas without the need of introducing extra components in the setup or leveling the amplitude of the reference and antenna branches, as it happens in conventional setups.

The increment in the separation of the image terms is achieved artificially without the need of physically increase the distance between the AUT and the reference antenna. This allows to reduce the size of the setup, regarding the size and distance of the acquisition plane, consequently decreasing the scan errors and the system sensitivity.

In case of maintaining the size of the acquisition plane, it has been demonstrated that the error of the retrieved signal is lowered on the edges of the acquisition plane with the proposed setup, allowing to increase the valid angular margin of the NF-FF transformation.

The use of spherical wave fronts as reference is also advantageous as the edge of the image terms can be controlled and the dynamic range of the hologram can be reduced as the image terms no longer have the shape of the AUT antenna [4]. 
To sum up, the presented method allows for an overlapping reduction of the terms of the hologram in the $k$-space, which eases the filtering process and improves the quality of the phase retrieval. The overlapping reduction is achieved thanks to the use of the replicas of the image terms and enables to apply this phaseless method even for the characterization of low-directivity antennas with wider spectra.

The main disadvantage of the proposed setup is that the sampling rate has to be slightly increased, as it happens with the synthesized reference setups, to be able to increment the extension of the spectral domain and displace the image terms to the non visible part of the spectrum.

Acknowledgments This work has been partially supported by the European Science Foundation under the RPN NEWFOCUS programme; By the Ministerio de Ciencia Innovación of Spain/FEDER under projects MIRIIEM-TEC 2014-54005-P and TEC 2014-55290-JIN; by the Gobierno del Principado de Asturias through the PCTI2013-2017, GRUPIN14-14 and by predoctoral grant BP11-169; by LINE525-002 grant and by the Ministerio de Economía y Competitividad under postdoctoral fellowship FPDI-2013-16278, and by the Academy of Finland through DYNAMITE project.

Open Access This article is distributed under the terms of the Creative Commons Attribution 4.0 International License (http://creativecommons.org/licenses/by/4.0/), which permits unrestricted use, distribution, and reproduction in any medium, provided you give appropriate credit to the original author(s) and the source, provide a link to the Creative Commons license, and indicate if changes were made.

\section{Appendix}

A plane reference wave can be defined, considering only the $x$-axis of the acquisition plane as

$$
E_{r}=A_{r} e^{-j \frac{\Delta \phi}{\Delta x} x}
$$

being $A_{r}$ the amplitude of the reference wave and $\Delta \phi$ and $\Delta x$ defining the phase and the sampling rate of the reference wave in the acquisition plane.

Accordingly, the expression of the sub-sampled signals (interspersed with zeros) corresponding to the even and odd samples of $E_{r}$ is given by

$$
\begin{gathered}
E_{r}^{(+)}(n P \Delta x)=\sum_{n} E_{r}(n P \Delta x) \delta(x-n P \Delta x) \\
E_{r}^{(-)}(n P \Delta x)=-\sum_{n} E_{r}(n P \Delta x) \delta(x-n P \Delta x-\Delta x)
\end{gathered}
$$

wherein $N$ is the number of samples and $P$ is the sub-sampling factor. Equation 12 includes the sign "-_" due to the $180^{\circ}$ phase shift although the change in the amplitude of the signal has been neglected. The reference signal used in the final processing of the hologram is the combination of the signals defined in Eqs. 11 and 12 and can be expressed as

$$
E_{r}^{(m)}(n P \Delta x)=\sum_{n} E_{r}(n P \Delta x) \delta(x-n P \Delta x)-\sum_{n} E_{r}(n P \Delta x) \delta(x-n P \Delta x-\Delta x)
$$

where the superscript $(m)$ indicates the multiplexing of both signals. Considering $P=2$, the term inside the brackets can be expressed as a Dirac comb function with 


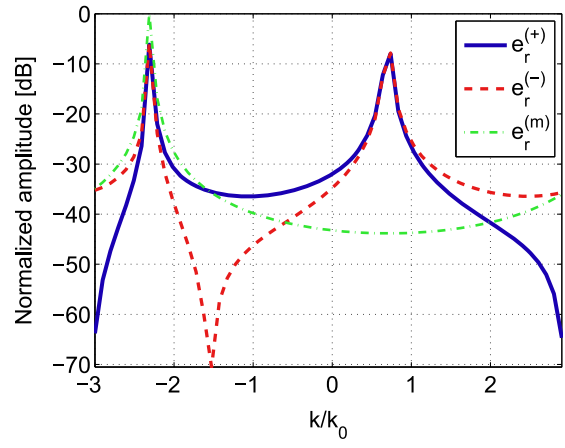

(a)

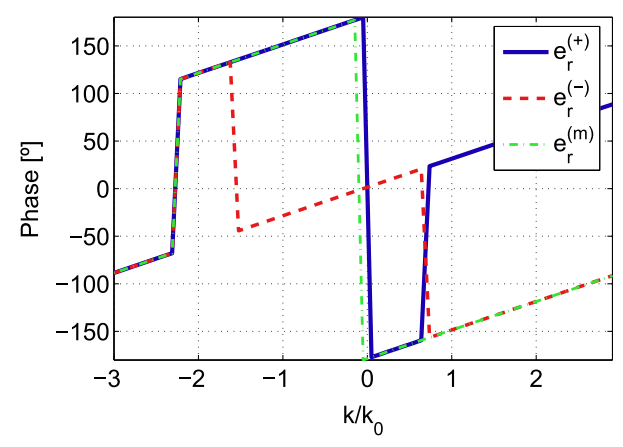

(b)

Fig. 10 Spectrum of the subsampled signals interspersed with zeros and the resulting multiplexed. a Normalized amplitude and $\mathbf{b}$ phase

alternating signs for each element and therefore, the expression in Eq. 13 can be rewritten as

$$
E_{r}^{(m)}(n \Delta x)=\sum_{n} E_{r}(n \Delta x) e^{-j \frac{\pi}{\Delta x} x} \delta(x-n \Delta x)
$$

and substituting Eq. 10 in Eq. 14 the reference signal becomes

$$
E_{r}^{(m)}(n \Delta x)=A_{r} e^{-j \frac{\Delta \phi-\pi}{\Delta x} x} \sum_{n} \delta(x-n \Delta x)
$$

and its FT corresponds to the following expression:

$$
e_{r}^{(m)}(k)=\widetilde{E_{r}}(n \Delta x)=\frac{A_{r}}{k_{s}} \sum_{n} \delta\left(k-n k_{s}+\frac{\Delta \phi-\pi}{\Delta x}\right)
$$

being $k_{s}$ the period of the spatial frequency domain defined as $1 / 2 \Delta x$.

The spectrum of the signals defined in Eqs. 11 and 12 should have replicas every $k_{s} / 2$ due to the sub-sampling in the spatial domain, while in Eq. 16 there is only one term of the reference signal per period placed on $k=-(\Delta \phi-\pi) / \Delta x+n k_{s}$. It can be concluded that for a perfect plane wave, the original terms of the spectrum are canceled due to the phase shift while the replicas are reinforced.

An example for the case of considering the spectrum of two plane waves as defined in Eqs. 11 and 12 with phase difference between consecutive samples of $45^{\circ}$ in the acquisition plane, and the signal obtained after multiplexing them, as defined in Eq. 16, is shown in Fig. 10. The sampling rate is $\lambda / 6$ for the multiplexed signal, while the original plane waves are sampled at $\lambda / 3$ and interspersed with zeros. The original terms are centered in $0.75 k_{0}$ (see Eq. 3), while the replica appears at a distance of $k_{s} / 2=3 k_{0}$, that is, at $-2.25 k_{0}$. It is clearly seen that, due to the phase shift of $180^{\circ}$ between both plane waves around $0.75 k_{0}$, the original terms are completely canceled as shown in Fig. 10b, and only the replica in $-2.25 k_{0}$ remains. 


\section{References}

1. A. Räisänen, J. Ala-Laurinaho, A. Karttunen, J. Mallat, A. Tamminen, M. Vaaja, 4th European Conference on Antennas and Propag. (EuCAP), pp. 1-3 (2010).

2. A. Yaghjian, IEEE Trans. Antennas Propag. 34(1), 30 (1986).

3. T. Huang, J. Bennett, G. Junkin, 9th International Conf. on Antennas and Propag., (Conf. Publ. No. 407) pp. 41-44 vol.1 (1995).

4. G. Junkin, T. Huang, J. Bennett, IEEE Trans. on Antennas Propag. 48(3), 409 (2000).

5. D. Gabor, Proc. of the Royal Soc. of London A: Math., Physical and Eng. Sci. 197(1051), 454 (1949).

6. E.N. Leith, J. Upatnieks, Journal of the Optical Soc. of America 52(10) 1123 (1962).

7. J. Bennett, A. Anderson, P. McInnes, A. Whitaker, IEEE Trans. on Antennas and Propag. 24(3) (1976).

8. A. Anderson, Proc. of the Institution of Electrical Engineers, 124(11), 946 (1977).

9. V. Schejbal, J. Pidanic, V. Kovarik, D. Cermak, IEEE Antennas Propag. Mag. 50(6), 89 (2008).

10. A. Olver, Antennas and Propagation, 1991. ICAP 91. Seventh International Conference on (IEE) 1, 99 (1991).

11. D. Smith, S. Skobelev, M. Leach, 8th International Symposium on Antennas, Propag. and EM Theory, 2008. ISAPE 2008 pp. 46-49 (2008).

12. V. Schejbal, V. Kovarik, D. Cermak, IEEE Antennas Propag. Mag. 50(5), 71 (2008).

13. J. Laviada Martinez, A. Arboleya-Arboleya, Y. Álvarez Lopez, C. García-González, F. Las-Heras, IEEE Antennas Wireless Propag. Lett. 13, 4 (2014).

14. D. Smith, M. Leach, M. Elsdon, S. Foti, IEEE Antennas and Propagation Magazine 49, 54 (2007).

15. D. Smith, M. Leach, S. Dlay, W. Woo, 6th International Symposium on Antennas, Propag. and EM Theory pp. 373-376 (2003).

16. D. Smith, M. Leach, A. Sambell, IEEE Microw. and Wireless Compon. Lett. 13(9), 379 (2003). 